\title{
Wavefront Detection from Intra-Atrial Recordings
}

\author{
U Richter ${ }^{1}$, M Stridh ${ }^{1}$, D Husser ${ }^{2}$, \\ DS Cannom ${ }^{3}$, AK Bhandari ${ }^{3}$, A Bollmann ${ }^{2}$, L Sörnmo $^{1}$ \\ ${ }^{1}$ Signal Processing Group, Dept of Electrical and Information Technology, Lund University, Sweden \\ ${ }^{2}$ Dept of Electrophysiology, Heart Center, University Leipzig, Germany \\ ${ }^{3}$ Dept of Cardiology, Good Samaritan Hospital and Harbor-UCLA Medical Center, Los Angeles, CA, \\ USA
}

\begin{abstract}
The present study deals with detection of intra-atrial wavefronts from atrial activation times in adjacent bipolar electrograms. A statistic of the delays within each wavefront was calculated and served as a basis for quantifying the wavefront consistency as well as the propagation of the electrical activity along the catheter. The database consisted of 19 patients for which five electrograms were recorded simultaneously during $10 \mathrm{~s}$. The analysis resulted in $38 \pm 2$ complete wavefronts per patient, i.e., wavefronts consisting of one activation from each recording site. Two parameters were evaluated for quantifying wavefront consistency, which together with the propagation profile well reflect the overall wavefront timing. In most cases, electrical activity was observed first in the high septal right atrium, and then spread along the catheter.
\end{abstract}

\section{Introduction}

Studies that quantify AF organization and synchronization, involving the detection of atrial activation times, have been presented previously [1-3]. A common approach is to base the calculations on coupling the activations of two adjacent recording sites, and to evaluate the time delay between each pair. These methods are often used to generate a map over the atria which reveals areas differing in their level of organization or synchronization.

In this study, the approach is quite similar, but the emphasis is on grouping the activations from more than two recording sites to wavefronts in order to evaluate the overall timing of the atrial activity recorded along the catheter. The results are expressed in terms of wavefront consistency during the recording as well as the propagation profile. Knowledge about which paths the wavelets take could provide help in guiding catheter ablations, aiming to terminate wavelet re-entry and by that the perpetuation of AF.

\section{Methods}

The method of the present study is structured into three analysis blocks, which are illustrated in the block diagram in Fig. 1. In the first step, the five recorded bipolar electrograms are preprocessed, resulting in detected activations for each bipolar electrogram. The activations serve as input to the wavefront detection, whose results are finally evaluated in the wavefront analysis.

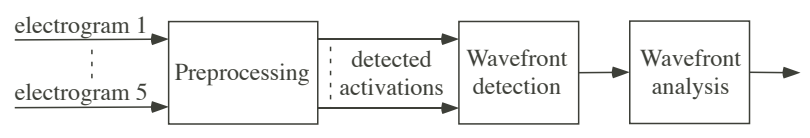

Figure 1. Block diagram of the method.

\subsection{Database}

The method was evaluated on a database of 19 patients with drug-refractory AF who underwent pulmonary vein catheter ablation and presented in $\mathrm{AF}$ at the begin of the procedure. Electrophysiological atrial studies were performed using a dual decapolar catheter (Cordis Webster Deflectable Halo) that was placed in the right atrium with the most distal pole (1) in the low lateral right atrium, poles 7 and 8 in the high lateral right atrium and poles 13 and 14 in the high septal right atrium. Ten bipolar electrodes were possible to measure $(\mathrm{H} 1, \mathrm{H} 3, \ldots, \mathrm{H} 19)$. In each patient the five adjacent bipolar electrodes that showed the most evident activity were chosen. In 14 patients, these were $\mathrm{H} 5$ to $\mathrm{H} 13$, in two patients $\mathrm{H} 7$ to H15, in another two patients $\mathrm{H} 9$ to $\mathrm{H} 17$ and in one patient $\mathrm{H} 11$ to $\mathrm{H} 19$. All recordings were sampled with $1 \mathrm{kHz}$ and were of 10 seconds duration.

\subsection{Preprocessing}

The requirements for wavefront detection are sufficient quality of the recording for reliable activation detection 
and that the activations in the different bipolar electrograms occur with approximately the same frequency under the investigated period of time. In order to determine the recording quality as well as the frequency dispersion, timefrequency characterization was applied [4] and resulted in one frequency spectrum per second for each bipolar electrogram. For quality rating, the position of the main peak in the 3 to $9 \mathrm{~Hz}$ range, as well as the power of this peak, was calculated for each spectrum. In case the power of the peak did not exceed $50 \%$ of the total power, the $1 \mathrm{~s}$ segment was excluded from further analysis. In case $50 \%$ or more of the total duration was excluded, the bipolar electrogram was not included in further analysis. Furthermore, those $1 \mathrm{~s}$ segments during which the standard deviation of the peak frequencies exceeded $0.5 \mathrm{~Hz}$ were excluded.

For activation detection, the atrial signals were preprocessed with bandpass filtering $(40-250 \mathrm{~Hz}$, order 40 , Kaiser window), rectification and low-pass filtering (FIR, 0-20 Hz, order 40, Kaiser window) [5-7]. Afterwards threshold detection was used to find the activation times. The threshold started at the time point of the last detected activation and was adapted to the amplitudes of previous detections by exponential averaging. Additionally, a blanking period of $50 \mathrm{~ms}$ was introduced in order to prevent multiple detections.

\subsection{Wavefront detection}

The presented method is based on processing the activations of two adjacent bipolar electrograms at a time, as it has been shown that the correlation between different recording sites decreases with distance [5]. For the first processed pair of bipolar electrograms, the matrix $\mathbf{W}$ with the wavefronts is initialized with one row for each bipolar electrogram, and the $N_{1}$ activation times of the first bipolar electrogram in the first row:

$$
\mathbf{W}=\left[\begin{array}{llll}
t_{1,1} & t_{1,2} & \cdots & t_{1, N_{1}} \\
0 & 0 & \cdots & 0 \\
\vdots & \vdots & & \vdots \\
0 & 0 & \cdots & 0
\end{array}\right]
$$

Next, the activations of the second bipolar electrogram are processed in a loop according to the following rule: The $i$ th activation of the $j$ th bipolar electrogram is added in $\mathbf{W}$ at column $k$ that yields

$$
\min _{k}\left(\left|t_{j, i}-t_{j-1, k}\right| \leq 90 m s\right), \quad j>1,
$$

i.e., the maximal allowed time difference between two activations is set to $90 \mathrm{~ms}$ [7]. In case the second bipolar electrogram has more activations than the first, those activations are added in a separate column on the appropriate place in the matrix, e.g.

$$
\mathbf{W}=\left[\begin{array}{llllll}
t_{1,1} & t_{1,2} & 0 & t_{1,3} & \cdots & t_{1, N_{1}} \\
t_{2,1} & t_{2,2} & t_{2,3} & t_{2,4} & \cdots & t_{2, N_{2}} \\
0 & 0 & 0 & 0 & \cdots & 0 \\
\vdots & \vdots & & \vdots & & \\
0 & 0 & 0 & 0 & \cdots & 0
\end{array}\right]
$$

The remaining bipolar electrograms are processed in a similar manner. After processing all bipolar electrograms, wavefronts that do not contain one activation from each recording site are removed. The wavefront matrix $\mathbf{W}$ then contains the activation times of the complete wavefronts in one column each, or, alternatively, the activation times of each bipolar electrogram which belong to a complete wavefront in one row each:

$$
\mathbf{W}=\left[\begin{array}{c}
\mathbf{t}_{1} \\
\mathbf{t}_{2} \\
\vdots \\
\mathbf{t}_{5}
\end{array}\right]
$$

\subsection{Wavefront analysis}

The wavefront analysis is done by measuring the consistency of the wavefronts during the recording time, as well as measuring the propagation profile, which investigates how the electrical activity is propagated along the catheter.

Wavefront consistency is based on the delays within the wavefronts:

$$
\delta_{\mathbf{n m}}=\mathbf{t}_{\mathbf{n}}-\mathbf{t}_{\mathbf{m}}
$$

where $n=1, \ldots, 4, m=n+1, \ldots, 5$, i.e., one delay vector is calculated for each possible combination of bipolar electrograms. For each delay vector $\delta_{\mathbf{n m}}$ the interquartile range $\bar{\delta}_{n m}$ was calculated, resulting in the matrix

$$
\overline{\boldsymbol{\Delta}}=\left[\begin{array}{llll}
\bar{\delta}_{12} & \bar{\delta}_{13} & \bar{\delta}_{14} & \bar{\delta}_{15} \\
0 & \bar{\delta}_{23} & \bar{\delta}_{24} & \bar{\delta}_{25} \\
0 & 0 & \bar{\delta}_{34} & \bar{\delta}_{35} \\
0 & 0 & 0 & \bar{\delta}_{45}
\end{array}\right] .
$$

Two strongly coupled recording sites will result in two bipolar electrograms whose activations occur with a consistent delay to each other, and thus a delay vector with low IQR. The opposite, i.e., a delay vector with high IQR, applies to two uncoupled recording sites. This fact is used to define the consistency parameter $C_{I Q R}$, which is calculated as the mean of the non-zero values of matrix $\bar{\Delta}$ and is measured in ms. Additionally, a normalized consistency measure ranging between 0 (inconsistent) and 1 (consistent) is desirable. This measure is based on the estimation of the Shannon entropy $S E$ [2]:

$$
C_{E, n m}=1-\frac{\hat{S E}}{\ln N},
$$


where $N$ is the number of detected wavefronts. Similar to the interquartile range, $C_{E, n m}$ is calculated for each delay vector $\delta_{\mathbf{n m}}$. Afterwards, the normalized wavefront consistency, $C_{E}$, for the complete recording is determined as the mean of the calculated values.

The propagation profile $P$ is based on the calculation of the medians $\tilde{\mu}_{n m}$ of the delay vectors, which can be summarized in a matrix:

$$
\tilde{\mathbf{M}}=\left[\begin{array}{lllll}
0 & \tilde{\mu}_{12} & \tilde{\mu}_{13} & \tilde{\mu}_{14} & \tilde{\mu}_{15} \\
-\tilde{\mu}_{12} & 0 & \tilde{\mu}_{23} & \tilde{\mu}_{24} & \tilde{\mu}_{25} \\
-\tilde{\mu}_{13} & -\tilde{\mu}_{23} & 0 & \tilde{\mu}_{34} & \tilde{\mu}_{35} \\
-\tilde{\mu}_{14} & -\tilde{\mu}_{24} & -\tilde{\mu}_{34} & 0 & \tilde{\mu}_{45} \\
-\tilde{\mu}_{15} & -\tilde{\mu}_{25} & -\tilde{\mu}_{35} & -\tilde{\mu}_{45} & 0
\end{array}\right] .
$$

To each row in matrix $\tilde{\mathbf{M}}$, a line is fitted. The quality of the fit is evaluated by calculating $r^{2}$, which can take values between 0 and 1 . A value close to 1 indicates that a greater proportion of variance is accounted for by the fitted line. The mean of the resulting $r^{2}$ values defines the propagation profile $P$ for each recording.

\section{Results}

After preprocessing, two bipolar electrograms were completely excluded. Of the remaining bipolar electrograms, $7.4 \pm 1.7 \mathrm{~s}$ (mean \pm standard deviation) were included in the analysis. Wavefront detection resulted in $38 \pm 2$ complete detected wavefronts per recording.

In the following, the method will be illustrated on two recordings shown in Figs. 2(a) and (b). The corresponding detected activation times and detected wavefronts are plotted in Figs. 2(c) and (d), respectively. For the example in the left column of Fig. 2, the electrograms as well as the detected wavefronts show a clear shift in the activation order from early H13 to late H13. The inconsistency of the wavefronts is well reflected by $C_{I Q R}$, which for the example is large with $40.6 \mathrm{~ms}$, and $C_{E}$, which is low with 0.28 . The example shown in the right column of Fig. 2 instead has a consistent wavefront pattern, which is reflected by the consistency measures $C_{I Q R}$ and $C_{E}$ that yield $14.1 \mathrm{~ms}$ and 0.5 , respectively.

Furthermore, the first example has a high propagation profile $(0.95)$, whereas in the second example $P$ has 0.21 . In the median, the electrical activity in the first example is propagating along the catheter, activating the different recording sites one after the other. This is not the case in the second example, where the first activation consistently occurs in the high septal right atrium at H13, and then propagates to $\mathrm{H} 11$ as well as $\mathrm{H} 15$.

The combination of both wavefront consistency and propagation profile reveals that, in the first example, the electrical activity is propagating, in median, along the catheter. However, there are large variations during the

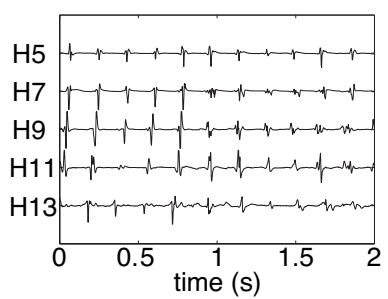

(a)

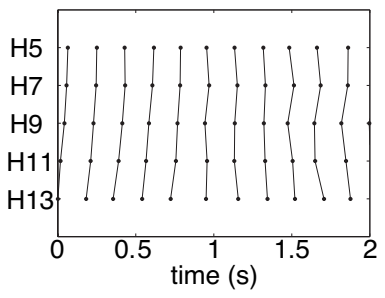

(c)

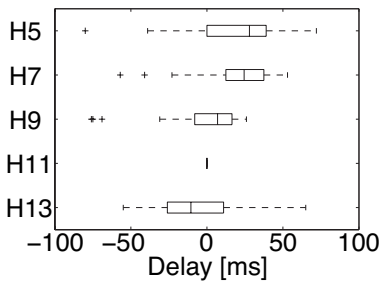

(e)

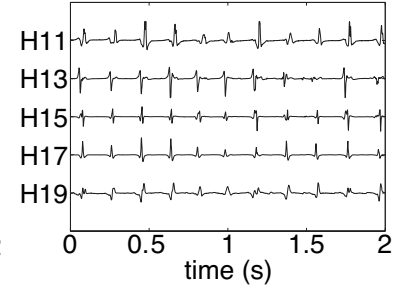

(b)

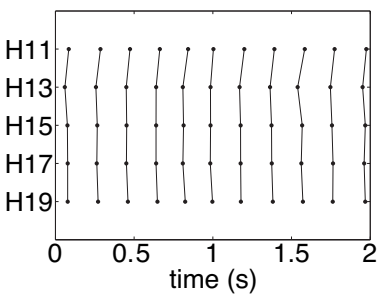

(d)

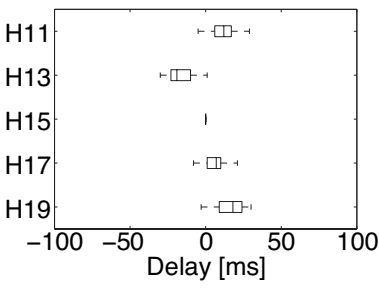

(f)
Figure 2. (a),(b) Two examples showing $2 \mathrm{~s}$ of the original bipolar electrograms. (c),(d) The corresponding detected activation times (dots) together with the detected wavefronts (lines). (e),(f) Box plots of the delay vectors with respect to $\mathrm{H} 11$ (e) and $\mathrm{H} 15$ (f).

recording time of $10 \mathrm{~s}$. In contrast, the electrical activity is not moving exactly along the catheter in the second example, but the variation in the wavefront pattern during the recording of $10 \mathrm{~s}$ is very low.

A possibility to visualize both wavefront consistency and propagation profile are box plots of the delay vectors, see Figs. 2(e) and (f). The bipolar electrograms H11 and $\mathrm{H} 15$ are chosen as reference for the first and the second example, respectively. The choice of reference has little influence as the difference between two medians $\tilde{\mu}_{n m_{1}}$, $\tilde{\mu}_{n m_{2}}$, where $m_{1} \neq m_{2}$, is $1.4 \pm 1.2 \mathrm{~ms}$. A comparison of the median wavefronts from Figs. 2(e) and (f) with those in Figs. 2(c) and (d) show that the median wavefront of the first example reflects only some of the wavefronts well, which is to be expected because of the high IQRs. Correspondingly, the median wavefront in the second example reflects the most wavefronts well, which is to be expected because of the low IQRs.

The results of the parameters $C_{I Q R}$ and $C_{E}$ for the complete database are summarized in Fig. 3(a), which clearly illustrates the implicit relation that a higher spread of the 


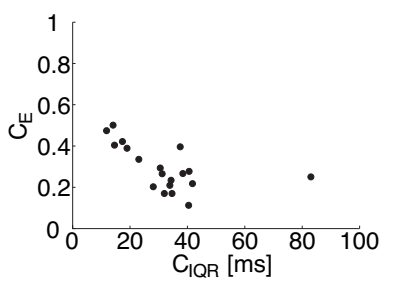

(a)

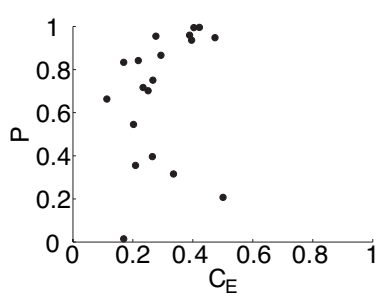

(b)
Figure 3. (a) Scatter plot of $C_{I Q R}$ vs. $C_{E}$. (b) Scatter plot of $C_{E}$ vs. $P$.

delays is connected with a high entropy, and thus low $C_{E}$. The statistics are $31.9 \pm 15.7 \mathrm{~ms}$ for $C_{I Q R}$ and $0.29 \pm 0.11$ for $C_{E}$. The propagation profile $P$ has for the complete database the statistics $0.68 \pm 0.30$. A scatter plot of $C_{E}$ and $P$ can be seen in Fig. 3(b), suggesting that the two parameters are uncorrelated.

\section{Discussion and conclusions}

As opposed to other studies where atrial activity is analyzed with the aim to distinguish between areas with different levels of organization or synchronization within the atria [1-3], this study is aiming to analyze the overall timing of the electrical activity along the catheter. It has been illustrated on two examples that both evaluated consistency parameters well reflect the consistency of the wavefronts over time. The two parameters differ such that one is quantifying the consistency resulting in a measure in $\mathrm{ms}$, while the other one is a normalized measure ranging between 0 and 1 . The results suggested a high correlation between the two parameters, of which thus one may be preferred depending on the present context.

The parameter propagation profile was introduced as a measure quantifying the propagation of the electrical activity along the catheter. As it is based on what can be interpreted as the median wavefronts of each recording, calculated for each bipolar electrogram as reference, it is important to also take the wavefront consistency into account: In case of high wavefront consistency the result of the propagation profile can be assumed to be valid for the majority of the wavefronts. A propagation profile close to 1 can be interpreted such that the first activation occurs in the most proximal/distal recorded bipolar electrogram, and moves then along the catheter. On the other hand, a propagation profile with a low value can mean that the first activation occurs in an bipolar electrogram in the middle of the recording region, and then spreads out along the catheter, or that there is no distinct activation pattern at all.

One limitation of this method is the need of reliable detection of atrial activation times, implying an increasing difficulty with decreasing organization of AF.
In conclusion, this study presented a method to detect intra-atrial wavefronts, as well as parameters which in a robust manner describe the propagation of the electrical activity along the catheter and the consistency of the wavefronts during the recording. In most cases, electrical activity was recorded first in the high septal right atrium, and then spread along the catheter. In the current study, five bipolar electrograms from a Halo catheter were included. The developed method is not restricted to this number of bipolar electrograms, but can easily be adapted to a larger number as well as multiple catheters.

\section{Acknowledgements}

D. Husser and M. Stridh are supported by the Volkswagen-Foundation. This study has been performed in part within the NordForsk network "Electrocardiology in Atrial Fibrillation".

\section{References}

[1] Barbaro V, Bartolini P, Calcagnini G, Censi F, Michelucci A. Measure of synchronisation of right atrial depolarisation wavefronts during atrial fibrillation. Med Biol Eng Comp 2002;40(1):56-62.

[2] Masè M, Faes L, Antolini R, Scaglione M, Ravelli F. Quantification of synchronization during atrial fibrillation by shannon entropy: Validation in patients and computer model of atrial arrhythmias. Physiol Meas 2005;26:911-923.

[3] Faes L, Ravelli F. A morphology-based approach to the evaluation of atrial fibrillation organization. IEEE Eng Med Biol Magazine 2007;59-67.

[4] Stridh M, Sörnmo L, Husser D, Bhandari AK, Cannom DS, Bollmann A. Time-frequency characterization of simultaneous intra-atrial and ecg recordings during atrial fibrillation. In Proc. Computers in Cardiology. 2005; .

[5] Botteron D, Smith J. A technique for measurement of the extent of spatial organization of atrial activation during atrial fibrillation in the intact human heart. IEEE Trans Biomed Eng 1995;42(6):579-586.

[6] Barbaro V, Bartolini P, Calcagnini G, Morelli S, Michelucci A, Gensini G. Automated classification of human atrial fibrillation from intraatrial electrograms. Pacing Clin Electrophysiol 2000;23:192-202.

[7] Faes L, Nollo G, Antolini R, Gaita F, Ravelli F. A method for quantifying atrial fibrillation organization based on wavemorphology similarity. IEEE Trans Biomed Eng 2002; 49(12):1504-13.

Address for correspondence:

Ulrike Richter

Dept. of Electrical and Information Technology, Lund University Box 118, SE-22100 Lund

ulrike.richter@eit.lth.se 